\title{
Vortex simulations of wind turbines operating in atmospheric conditions using a prescribed velocity-vorticity boundary layer model
}

Ramos García, Néstor; Spietz, Henrik Juul; Sørensen, Jens Nørkær; Walther, Jens Honore

Published in:

Wind Energy

Link to article, DOI:

$10.1002 /$ we.2225

Publication date:

2018

Document Version

Peer reviewed version

Link back to DTU Orbit

Citation (APA):

Ramos García, N., Spietz, H. J., Sørensen, J. N., \& Walther, J. H. (2018). Vortex simulations of wind turbines operating in atmospheric conditions using a prescribed velocity-vorticity boundary layer model. Wind Energy, 21(11), 1216-1231. https://doi.org/10.1002/we.2225

\section{General rights}

Copyright and moral rights for the publications made accessible in the public portal are retained by the authors and/or other copyright owners and it is a condition of accessing publications that users recognise and abide by the legal requirements associated with these rights.

- Users may download and print one copy of any publication from the public portal for the purpose of private study or research.

- You may not further distribute the material or use it for any profit-making activity or commercial gain

- You may freely distribute the URL identifying the publication in the public portal 


\title{
RESEARCH ARTICLE
}

\section{Vortex simulations of wind turbines operating in atmospheric conditions using a prescribed velocity-vorticity boundary layer model}

\author{
Néstor Ramos-García ${ }^{1}$, Henrik Juul Spietz ${ }^{2}$, Jens Nørkær Sørensen ${ }^{1}$, and Jens Honoré Walther ${ }^{2,3}$ \\ ${ }^{1}$ Department of Wind Energy, Building 403, Technical University of Denmark, DK-2800 Kgs. Lyngby, Denmark \\ 2 Department of Mechanical Engineering, Building 403, Technical University of Denmark, DK-2800 Kgs. Lyngby, Denmark \\ ${ }^{3}$ Computational Science and Engineering Laboratory, ETH Zürich, Clausiusstrasse 33, CH-8092 Zürich, Switzerland
}

\begin{abstract}
A prescribed velocity-vorticity boundary layer model for the vorticity transport equation is proposed, which corrects the unphysical upward deflection of the wake seen in a simpler prescribed velocity shear approach. A Lagrangian implementation of the boundary layer model has been investigated using our in-house vortex solver MIRAS. The MIRAS code contains both an aerodynamic part and an structural-mechanical part taking into account aeroelastic phenomena. The solver is employed to simulate flows around wind turbines and uses a combination of filaments and particles in order to mimic the vorticity released by the wind turbine blades. The vorticity is interpolated onto a uniform Cartesian mesh, where the interaction is efficiently calculated by an FFT-based method. Simulations of wind turbines operating in an atmospheric boundary layer flow are carried out and analyzed in detail for a range of scenarios. The manuscript focuses on studying the influence of wind shear and turbulence, which is varied to mimic natural atmospheric conditions. A traverse virtual probe up to 30 diameters downstream of the rotor plane is used to investigate the properties of the turbulent wake flow for the different cases. This includes mean and standard deviation of the stream-wise velocity component, wake deficit, Reynolds stresses and power spectral density of the velocity signal. The results show that combining a prescribed boundary layer approach with a vortex method gives consistent and physically correct results if properly implemented. Copyright (c) 2013 John Wiley \& Sons, Ltd.
\end{abstract}

\section{KEYWORDS}

Atmospheric boundary layer; Wind shear; Turbulence; Wind turbine; Vortex Method; Aeroelasticity

\section{Correspondence}

Department of Wind Energy, Fluid Mechanics Section, Building 403, Technical University of Denmark, DK-2800 Lyngby Denmark. E-mail: nerga@dtu.dk

\section{Contract/grant sponsor}

FP7-ENERGY-2013-1

Contract/grant number

608396

Received... 


\section{INTRODUCTION}

Vortex methods initially entered the wind energy community as a more physically accurate alternative to the basic Blade Element Momentum method (BEM) technique, which is based on one-dimensional momentum theory and aimed to be used for rotor design. However, vortex methods have quickly developed in recent years, and now can be seen as an advanced and matured tool capable of performing high-fidelity simulations of one or more turbines. Vortex methods are not only of interest during the actual rotor design process, but also as analysis tool to investigate more complex scenarios, including aeroelastic, turbulent inflow and shear effects [1-6].

Vortex solvers can be combined with different aerodynamic models depending on the degree of complexity required. Enumerated in ascending complexity, they can be classified as: lifting line, vortex lattice, potential panel method and viscous-inviscid panel method. The latter class being the more physically correct, since it resolves the actual geometry of the blade accounting for the viscous effects enclosed inside the boundary layer. The present paper focuses on the lower degree of fidelity in terms of aerodynamic modeling of the wind turbine blades, using the lifting line approach (LL). With the LL model, the rotor blades are represented by discrete filaments, which account for the bound vortex strength and release vorticity into the flow. This vorticity can be subdivided into two categories: trailing and shed vorticity. It is important to note here that, although this article focuses on the lower fidelity aerodynamic model, the proposed method can be used in combination with higher fidelity models in a similar fashion.

BEM [7], which at this point is considered as the industry standard, is not capable by its very nature to accurately simulate complex blade geometries such as winglets and flaps or blade curvature including sweep and prebend. The same occurs for complex flow cases, e.g. yaw, shear or even to account for the interaction between the incoming turbulence, the wind turbine and its wake. More or less simple model extensions are needed to take into account some of these scenarios, while others are impossible to reproduce. It is therefore necessary to develop higher-fidelity tools to simulate such cases and use the obtained knowledge to improve/calibrate the fast engineering tools. This necessity has been highlighted by the wind energy community in recent years [8]. Fully resolved CFD applied to wind turbine rotors has been developed for many years now, becoming a mature and trusted tool. However, a tool that operates in the range from low to high fidelity and give physical and accurate results at low computational costs is of great interest to the community. Vortex methods have the potential of becoming such a tool.

It is standard practice for the modeling of a wind shear in a free-wake vortex simulation to prescribe the velocity field seen by the vortex elements as function of the height. This velocity is added to the wake induction and used for the vortex element advection, as done in [9]. This approach seems to be capable of capturing the correct blade loadings, but it predicts an unphysical behavior of the wake by deflecting it upwards. This behavior limits the use of vortex methods in atmospheric conditions, and in particular makes it impossible to study the wake interaction between multiple turbines. Branlard et al. [10] proposed the idea of imposing, in addition to the prescribed velocity field, a prescribed vorticity field. In order to do that, the vorticity transport equation has to be re-written in terms of both free and prescribed velocity and vorticity. The authors concluded that his method improved, but not totally corrected the erroneous upward motion of the wake seen in the prescribed velocity implementation. They proposed another method where the shear was carried by a set of vortex particles released upstream of the wind turbine. This method was able to successfully simulate a light shear profile. In finite-volume solvers, a simplified boundary layer model using a distribution of prescribed body forces in combination with an actuator line model have previously been used in the wind energy community, see e.g. [11]. The prescribed approach, both in vortex methods and finite-volume solvers, can be seen as a simplified engineering approach to modeling the atmospheric boundary layer. However, the prescribed approach is extremely useful in order to perform parametric studies like those carried out by Andersen [12,13], as it allows full control of the different inflow characteristics. Such control can be key in engineering studies where a set of pre-defined or measured atmospheric conditions need to be reproduced as accurately as possible, cf. e.g. Hasager [14].

In this paper, we introduce a new prescribed velocity-vorticity boundary layer method (P2VBL) capable of modeling shear effects and wakes of wind turbines located in clusters. This new approach is able to cope with the steep velocity 
gradients inherent to atmospheric boundary layers near the ground, enabling it to simulate extreme shear cases. The turbulent fluctuations which are computed from a pre-generated turbulence field, are taken into account by releasing a set of vortex particles in a plane upstream of the wind turbine. The Manns turbulence model [15] is used to generate the relevant velocity field, which is transformed into a particle cloud and released upstream of the rotor plane as in [16].

The P2VBL model has been successfully implemented in the in-house solver MIRAS. MIRAS simulates the vortex shedding of wind turbine rotors using a sheet of vortex filaments which is transformed into a set of particles, using a hybrid filament-mesh wake [17]. The hybrid filament-mesh implementation is an extension of the MIRAS code with the high order mesh-based Poisson solver of Hejlesen et al. [18-20]. This extension significantly increases the computational efficiency while maintaining the accuracy of the original direct filament method. In the present work, the solver accounts for stretching and diffusive terms in the vorticity transport equation by using a finite difference approach in which the vorticity variations are computed directly on the mesh. An eight-order spatial discretization is used in view of an observed non-physical distortion of the cylindrical wake shape caused by the usage of low order stencils. A periodic re-meshing of the particles is employed to maintain a smooth field. The field is forced to remain divergence free by applying a reprojection of the vorticity field every time step.

The most important characteristics of the disturbed flow downstream a wind turbine are the wake deficit and the turbulence intensity (TI). The first one is directly related to the power extracted by the turbine, while the second one, enhanced by the interaction with the turbine wake is responsible of the blade loadings and therefore very important for the life-span design of a turbine. Both characteristics are key parameters when studying the effect of an upstream turbine on downstream located machines. It is hence important to asses the ability of the P2VBL model to capture the downstream development of such quantities. Unfortunately the access to high-quality measurements on full-size wind turbine wakes is limited. Therefore, in this paper, we are restricted to carry out a qualitative comparison to experimental work carried out under wind tunnel conditions and for small size rotors, [21, 22].

The paper is organized as follows. In Section 2, the hybrid vortex method and the prescribed velocity-vorticity boundary layer model are presented, including the lifting line aerodynamic model and the inflow turbulence modeling. The numerical results are presented in Section 3, which is divided into four subsections. In the first subsection, the general characteristics of the numerical setup are described. In subsections two and three, the presented shear and inflow turbulence models are compared with the standard-practice approach (i.e. velocity prescribed shear and frozen velocity turbulence box). In the last subsections, the P2VBL model is verified by doing a parametric study. Simulations are presented for various wind shear coefficients and incoming turbulent intensities where the different wake quantities are analyzed. Detailed analysis of wake quantities include mean and standard deviation of the stream-wise velocity, wake deficit, Reynolds stresses, and power spectral density of the velocity signal. Conclusions are summarized in Section 4.

\section{METHODOLOGY}

\subsection{Hybrid vortex method}

In the hybrid vortex method, the vorticity shed by the blades is initially carried by discrete vortex filaments in the form of shed and trailing vorticity, which further downstream are transformed into particles. The interaction between the vortex filaments is calculated by a direct Biot-Savart calculation, while the interaction of the particles is calculated by a meshbased method similar to the one originally proposed in [23].

In the present study, a lifting line technique is employed as the blade aerodynamic model. The blade bound circulation is modeled by a single vortex line, located at the blades quarter-chord and sub-divided in vortex segments, which represent the wind turbine blades. The vorticity is released into the flow by a row of vortex filaments following the chord direction (shed vorticity) and a row of filaments perpendicular to the chord direction (trailing vorticity) at the different blade sections. The first one accounts for the released vorticity due to the time variation of the bound vortex strength, while the second one accounts for the vorticity released due to circulation gradients along the span-wise direction of the blade. 


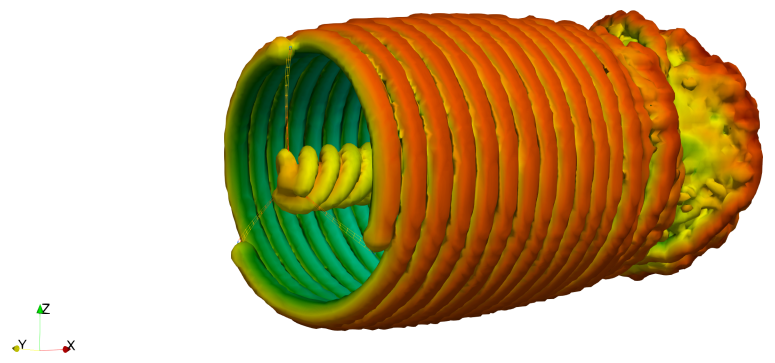

Figure 1. Visualization of the flow past a wind turbine rotor simulated with the hybrid filament-mesh method. The vorticity iso-contours are colored by the magnitude of the stream-wise velocity.

The strength of the bound vortex, $\Gamma$, is calculated applying the Kutta-Joukowsky theorem in each of the spanwise stations along the blade,

$$
\Gamma=\frac{L}{\rho V_{c / 4}}
$$

where $V_{c / 4}$ is the total velocity computed at the quarter chord of the different blade sections, and $L$ is the lift force at the same location. The lift is obtained directly by interpolation in a set of tabulated airfoil data $\left(C_{l}, C_{d}, C_{m}\right)$ as function of the computed angle of attack (AoA).

The length of the first row of filaments is calculated using the quarter chord velocity magnitude and re-calculated in a sub-iterative convergence loop within each time step. Convergence is attained when the residual, defined as the variation in AoA, is smaller than $10^{-4}$. A relaxation factor of 0.05 is used to ensure a smooth convergence. A maximum number of iterations is set to 50. The convergence rate is directly related to the unsteadiness of the problem, and convergence is usually attained within 2 to 10 iterations. The circulation of the vortex filaments remains constant in time with their motion being described by Lagrangian filament markers placed at their end points.

The downstream location of the transformation from filaments to particles depends on the problem specifications and is usually defined by the user. Optimization problems where large time steps are necessary could benefit from a longer filament-based wake, while cases like the one we analyze in this article, which include aeroelastic effects and turbulence, and where a small time-step is necessary, strongly benefit from a short filament-wake (less than 3 degrees in azimuthal rotation is usually employed). A visualization of the simulated flow past a wind turbine rotor using the hybrid method is shown in Figure 1. In the figure, the vortex filaments are visualized by a wire-frame representation, while further downstream the vorticity carried by the vortex particles is visualized using an iso-contour of their magnitude on the mesh. The trajectory of the vortex elements is determined by the velocity of their markers $\underline{u}\left(\underline{x}_{i}\right)$ (endpoints in the case of filaments or the point itself in the case of particles), which in turn is calculated by a superposition of the free-stream velocity $\underline{V}_{0}$ and the velocity contributions from all vortex and boundary elements at the marker positions $\underline{x}_{i}$,

$$
\frac{d \underline{x}_{i}}{d t}=\underline{u}\left(\underline{x}_{i}\right) \quad \text { with } \quad \underline{u}\left(\underline{x}_{i}\right)=\underline{V}_{0}+\underline{u}_{\Gamma}\left(\underline{x}_{i}\right)+\underline{u}_{\mathrm{fw}}\left(\underline{x}_{i}\right)+\underline{u}_{\mathrm{pw}}\left(\underline{x}_{i}\right)
$$

Here $\underline{u}_{\Gamma}$ is the velocity induced by the blade bound vortex, and $\underline{u}_{\mathrm{fw}}$ and $\underline{u}_{\mathrm{pw}}$ are the velocities induced by the vortex elements of the filament-wake and particle-wake regions, respectively. The calculation of the different velocity contributions of Equation (2) will be explained in detail in the following.

The bound vortex velocity contribution, $\underline{u}_{\Gamma}$, is obtained directly by evaluating the Biot-Savart law. Since this procedure can become computationally expensive for large domains with a high amount of vortex particles, the influence of the bound vortex is truncated at a distance of one diameter downstream of the rotor plane. Various simulations including and excluding the influence of the bound vortex have been performed for a single and for multiple turbines in a row, showing that differences both in terms of blade loadings and wake characteristics are negligible.

The filament-wake velocity contribution, $\underline{u}_{\mathrm{fw}}$, is obtained directly by evaluating the Biot-Savart law and summing up the velocity induced by all filaments that form the near wake. A regularized Biot-Savart law is applied by introducing a 


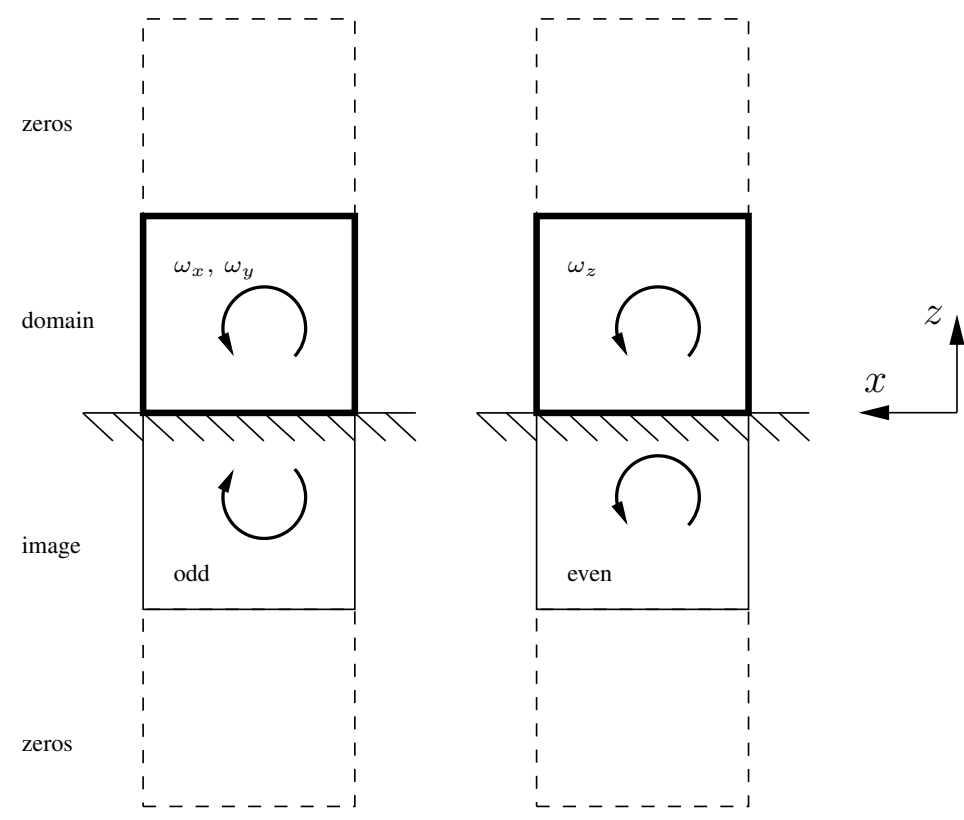

Figure 2. Symmetries assumed for the vorticity in order to achieve velocity boundary conditions on the slip plane.

finite vortex core to avoid a singular interaction between filaments. A Scully cut-off velocity profile, [24], has been used to model the vortex core. In the present work the near wake has been reduced to two rows of filaments, as can be seen in Figure 1, in order to speed up the simulations.

The particle-wake velocity contribution $\underline{u}_{\mathrm{pw}}$, is obtained by transforming the circulation of the filaments into a vorticity distribution on an auxiliary uniform Cartesian mesh where the interaction is efficiently calculated by an FFT-based method. The transformation from filaments to particle-mesh is done in two steps. First, the filaments are transformed into particles, and the integral vorticity vector of the generated particles is computed from the filament circulation $\Gamma$. Second, the generated particles are interpolated onto the mesh. Once the vorticity field has been constructed on the mesh, a Poisson equation is solved for the velocity by convolution with a free-space Green's function in Fourier space.

The singular free-space Green's function is regularised by a tenth-order Gaussian filter to obtain a higher order approximation following $[18,20]$. The width of the filter function is here chosen as $\varepsilon_{m}=1.5 h$ in order to minimize the smoothing error associated with the regularization, while resolving the filter function accurately. Thereby we obtain an effective kernel size which is close to that used in the direct filament-filament calculations.

The linear convolution is computed using FFTs with domain doubling following the procedure of Hockney and Eastwood [23]. Here we extend the procedure to include Neumann and Dirichlet conditions using the method of images.

In the current work we consider the half-unbounded problem with a no-through condition on the ground plane $(\{x, y, 0\})$

$$
\underline{u} \cdot \underline{n}=\underline{0}, \quad \frac{\partial \underline{u}}{\partial z} \cdot \underline{t}=\underline{0} \quad \text { on }\{x, y, 0\} .
$$

This is done by solving an extended problem as depicted in Figure 2, where we account for the vorticity field mirrored about the ground plane. For the first and second component we must account for the odd images and for the even image for the third component to impose the stated conditions. The domain symmetrization is implicitly done by replacing the discrete Fourier transform by a combination of even and odd discrete trigonometric transforms in the $z$-direction. Thereby the modification only imposes minor additional costs by using an efficient implementation based on the FFT and exploiting the symmetries in the data.

We note that a no-slip condition may be achieved by including a second substep, where a vortex sheet necessary to cancel the slip-velocity is computed and diffused into the domain, as in [25-27] 
For flows involving wind turbines in atmospheric boundary layers the Reynolds number is well beyond the point where direct numerical simulation is practical. Hence the current simulations must be regarded as large-eddy simulations since all scales will not be resolved. We apply an explicit filtering method as [28] that may be viewed as an approximate deconvolution model $[29,30]$ using a smooth filter $(\zeta)$ as the combined primary and approximately inverse filter. In the current work the filter is the same as the one used to solve the Poisson equation with high order accuracy. This ensures consistency between the dependent variables in the current formulation. We apply the filter once for every time step, according to the work of Stolz et al. [30], who used an explicit filter and found small sensitivity for a combined filter with spectral midpoint $\left(\hat{\zeta}\left(k_{c}\right)=0.5\right)$ at $k_{c} \approx 0.8 \pi$. For comparison, the transfer function of the tenth order Gaussian filter of width $1.5 \Delta x$ is $k_{c} \approx 0.65 \pi$.

\subsection{Atmospheric boundary layer model}

The implementation of the P2VBL model in a vortex solver will be introduced in the following. The vorticity equation, which is obtained by taking the curl of the Navier-Stokes equations, describes the evolution of the vorticity, $\underline{\omega}=\nabla \times \underline{u}$, of a fluid particle as it moves with the flow. Assuming an incompressible fluid with a constant and uniform viscosity, the vorticity equation is written as follows,

$$
\frac{D \underline{\omega}}{D t}=\frac{\partial \underline{\omega}}{\partial t}+(\underline{u} \cdot \nabla) \underline{\omega}=(\underline{\omega} \cdot \nabla) \underline{u}+\nu \nabla^{2} \underline{\omega}
$$

The left hand side of the equation describes the rate of change of the vorticity and it includes the unsteady term, $\frac{\partial \omega}{\partial t}$, and the convection term, $(\underline{u} \cdot \nabla) \underline{\omega}$, which accounts for the changes in vorticity due to the motion of the fluid particle. On the right hand side, $(\underline{\omega} \cdot \nabla) \underline{u}$, accounts for the vortex stretching and $\nu \nabla^{2} \underline{\omega}$ for the vortex diffusion due to viscous effects. The velocity and vorticity terms are divided in free $\left(\underline{u}^{\prime}, \underline{\omega}^{\prime}\right)$ and prescribed $\left(\underline{u}_{p b l}, \underline{\omega}_{p b l}\right)$ contributions,

$$
\underline{\omega}=\underline{\omega}^{\prime}+\underline{\omega}_{p b l}, \quad \underline{\omega}_{p b l}=\omega_{p b l}(z) \cdot \underline{e}_{y} \quad \text { and } \quad \underline{u}=\underline{u}^{\prime}+\underline{u}_{p b l}, \quad \underline{u}_{p b l}=u_{p b l}(z) \cdot \underline{e}_{x}
$$

Taking into account the above definitions, Equation 4 can be re-written as follows,

$$
\begin{gathered}
\frac{\partial\left(\underline{\omega}^{\prime}+\underline{\omega}_{p b l}\right)}{\partial t}+\left(\underline{u}^{\prime}+\underline{u}_{p b l}\right) \cdot\left(\nabla \underline{\omega}^{\prime}+\nabla \underline{\omega}_{p b l}\right)= \\
\left(\underline{\omega}^{\prime}+\underline{\omega}_{p b l}\right) \cdot \nabla\left(\underline{u}^{\prime}+\underline{u}_{p b l}\right)+\nu \nabla^{2}\left(\underline{\omega}^{\prime}+\underline{\omega}_{p b l}\right)
\end{gathered}
$$

As we use a particle-mesh method, where the particles vorticity is interpolated onto a mesh, the newly introduced convective terms related to the effect of shear can be computed both in an Eulerian or a Lagrangian manner. The Lagrangian approach utilizes the primary advantage of vortex-particle methods as the entire convection of the free vorticity is performed by advecting particles in the combined velocity field. This approximation introduces negligible numerical diffusion and has better stability properties than the corresponding Eulerian approximation and is therefore formally better suited. The Lagrangian approach offers an overall better behavior in terms of first and second order statistics of the wake flow, therefore being the focus of this manuscript. Following the Lagrangian implementation Equation 6 yields,

$$
\begin{array}{r}
\frac{D \underline{\omega}^{\prime}}{D t}=\frac{\partial \underline{\omega}^{\prime}}{\partial t}+\underline{u}^{\prime} \cdot \nabla \underline{\omega}^{\prime}+\underline{u}_{p b l} \cdot \nabla \underline{\omega}^{\prime}= \\
\underline{\omega}^{\prime} \cdot \nabla \underline{u}^{\prime}+\underline{\omega}^{\prime} \cdot \nabla \underline{u}_{p b l}+\underline{\omega}_{p b l} \cdot \nabla \underline{u}^{\prime}+\underline{\omega}_{p b l} \cdot \nabla \underline{u}_{p b l} \\
+\nu \nabla^{2} \underline{\omega}^{\prime}+\nu \nabla^{2} \underline{\omega}_{p b l}-\underline{u}^{\prime} \cdot \nabla \underline{\omega}_{p b l}
\end{array}
$$

Free vorticity convection is simply modeled by marching the fluid particles with the prescribed velocity as function of their height. However, as shown in Equation 7, the convection of the prescribed vorticity, i.e. $u_{z}^{\prime} \frac{\partial \omega_{p b l y}}{\partial z}$ term, which is not carried out by the free vortex particles, is accounted for in the right-hand side of the equations and computed using 
finite differences on the mesh together with the diffusive and the stretching terms. For a more detailed explanation of the influence of the added terms to the vorticity transport equation, Equation 7 is further expanded in each direction

$$
\begin{gathered}
\frac{\partial \omega_{x}^{\prime}}{\partial t}+\left(\underline{u}^{\prime}+\underline{u}_{p b l}\right) \cdot \nabla \omega_{x}^{\prime}=\omega_{x}^{\prime}\left(\frac{\partial u_{x}^{\prime}}{\partial x}\right)+\left(\omega_{y}^{\prime}+\omega_{p b l y}\right)\left(\frac{\partial u_{x}^{\prime}}{\partial y}\right)+ \\
\omega_{z}^{\prime}\left(\frac{\partial u_{x}^{\prime}}{\partial z}+\frac{\partial u_{p b l x}}{\partial z}\right)+\nu\left(\frac{\partial^{2} \omega_{x}^{\prime}}{\partial x^{2}}+\frac{\partial^{2} \omega_{x}^{\prime}}{\partial y^{2}}+\frac{\partial^{2} \omega_{x}^{\prime}}{\partial z^{2}}\right) \\
\frac{\partial \omega_{y}^{\prime}}{\partial t}+\left(\underline{u}^{\prime}+\underline{u}_{p b l}\right) \cdot \nabla \omega_{y}^{\prime}=\omega_{x}^{\prime}\left(\frac{\partial u_{y}^{\prime}}{\partial x}\right)+\left(\omega_{y}^{\prime}+\omega_{p b l y}\right)\left(\frac{\partial u_{y}^{\prime}}{\partial y}\right)+ \\
\omega_{z}^{\prime}\left(\frac{\partial u_{y}^{\prime}}{\partial z}\right)+\nu\left(\frac{\partial^{2} \omega_{y}^{\prime}}{\partial x^{2}}+\frac{\partial^{2} \omega_{y}^{\prime}}{\partial y^{2}}+\frac{\partial^{2} \omega_{y}^{\prime}}{\partial z^{2}}+\frac{\partial^{2} \omega_{p b l y}}{\partial z^{2}}\right)-u_{z}^{\prime} \frac{\partial \omega_{p b l y}}{\partial z} \\
\frac{\partial \omega_{z}^{\prime}}{\partial t}+\left(\underline{u}^{\prime}+\underline{u}_{p b l}\right) \cdot \nabla \omega_{z}^{\prime}=\omega_{x}^{\prime}\left(\frac{\partial u_{z}^{\prime}}{\partial x}\right)+\left(\omega_{y}^{\prime}+\omega_{p b l y}\right)\left(\frac{\partial u_{z}^{\prime}}{\partial y}\right)+ \\
\omega_{z}^{\prime}\left(\frac{\partial u_{z}^{\prime}}{\partial z}\right)+\nu\left(\frac{\partial^{2} \omega_{z}^{\prime}}{\partial x^{2}}+\frac{\partial^{2} \omega_{z}^{\prime}}{\partial y^{2}}+\frac{\partial^{2} \omega_{z}^{\prime}}{\partial z^{2}}\right)
\end{gathered}
$$

From Equation 8 it can be seen how the P2VBL terms contribute only to vortex stretching in the $x$ and $z$ direction. While in the $y$ direction the prescribed boundary layer terms also influence the convection and diffusion mechanisms.

\subsection{Inflow turbulence modelling}

The Mann model [15] is used to generate a synthetic turbulent velocity field on a uniform mesh (commonly known as a turbulence box) representative of land-based atmospheric conditions with a given turbulence intensity (TI). In order to feed this turbulent field into the computational domain, two approaches are considered. In the first approach, the actual turbulent box is displaced through the computational domain at a velocity equal to the free-stream velocity. The influence of turbulence on the rotor blades is calculated by using a tri-linear interpolation. This procedure is straightforward due to the constant mesh spacing used for the discretization of the turbulence box. However the influence of the turbine and its wake in the incoming turbulence is not taken into account. Therefore this approach we refer to as a frozen velocity inflow model. In the second approach, the velocity field is transformed into a vortex-particle cloud which is gradually released into the computational domain at a plane upstream the wind turbine, similar to the modeling of onocoming turbulence and associated admittance in bridge aerodynamics [31-33]. In the present work the vortex particles are released 2 diameters upstream of the rotor plane. We refer to this approach as a free vorticity inflow model. In a first step, the curl of the velocity field is computed to obtain the vorticity field. The strengths of the vortex-particles are obtained by multiplying the vorticity field with the local cell volume. In this way, one particle is created per cell in the turbulent box. These turbulent particles will freely interact with the complete flow when released. This approach is used in all the investigations included in the manuscript. However, a short verification against the frozen velocity-based approach (taken as the industry standard) is presented in Section 3.3.

\section{NUMERICAL RESULTS}

In the following, the proposed atmospheric boundary layer model is verified by investigating different scenarios.

\subsection{Computational mesh and simulation set-up}

The comparison between the different shear models is presented in the first subsection. For this study a mesh with an extent of $L_{x} \times L_{y} \times L_{z}=4 D \times 2 D \times 2 D$, where $D$ is the rotor diameter, and $L_{x}, L_{y}$ and $L_{z}$ is the streamwise, horizontal and vertical domain length, respectively. For the rest of the simulations presented in this section, a Cartesian mesh with $32 D \times 3 D \times 3 D$, as depicted by the sketch in Figure 3, is employed. A constant spacing of $0.7 \mathrm{~m}$, approximately 20 


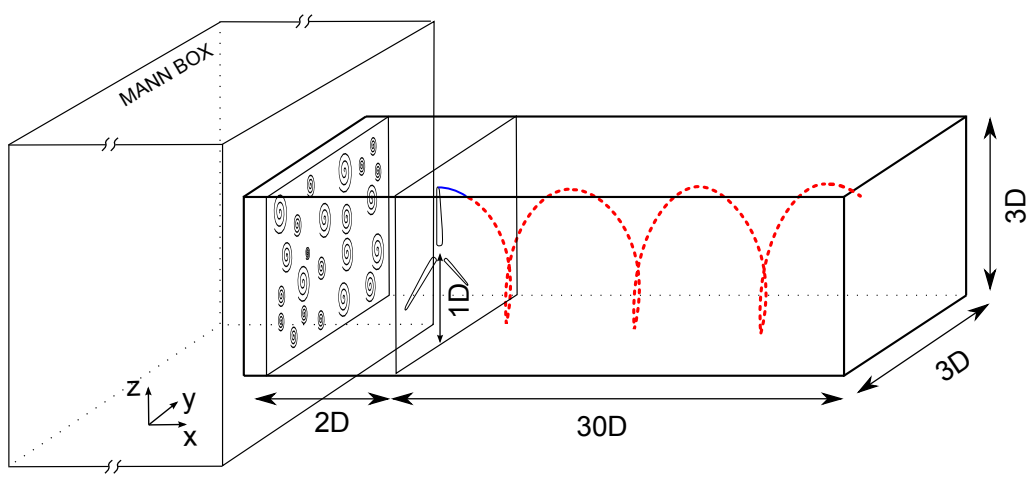

Figure 3. Sketch of the computational domain used in MIRAS simulations.

cells per blade, is used in all three directions, resulting in a mesh with $1335 \times 129 \times 129$ cells. This sums up to a total of more than 22 million cells with a number of vortex particles in the same order. The rotor center is placed approximately 1 diameter from the ground, centered around the $y$ direction and 2 diameters downstream of the turbulent inflow release location. A small time step of $0.01 \mathrm{~s}$ is fixed due to aeroelastic constrains. A total number of 50000 time steps have been simulated for all cases (unless stated otherwise). The analysis performed in the following sections use the data recorded for the last 30000 time steps.

The turbulent box used in all computations is much larger than the actual simulated domain, $1122 D \times 9 D \times 9 D$, in order to include large structures in the simulation. Moreover, the discretization of the box is coarser, constant spacing of $2 \mathrm{~m}$, which is around 3 times larger than the computational cells. In this way the smaller turbulent structures are generated/controlled by the solver.

A replica of the V29 VESTAS rotor with a diameter size of $29.2 \mathrm{~m}$ is used (private communication). The replica rotor blades have been designed using the NACA 63-4 airfoil family, with thicknesses ranging from $14 \%$ to $24 \%$ and a linear interpolation to the root where a cylinder section is used. The airfoil polars used in the simulations have been obtained by a combination of wind tunnel experiments in the low/mid-range of angles of attack and an analytical stall model for the high angle of attack region.

The wind profile power law, which gives an estimation of how the mean wind speed changes as function of the height in an atmospheric boundary layer is used to define the boundary layer shape. The use of this formula makes it possible to model different shear profiles in a generic way. It is important to remark that with the P2VBL model, multiple types of shear can be prescribed, for example from an experimental campaign. It is therefore a very versatile tool for investigation of field cases. The power law formula can be written as follows,

$$
u_{p b l}(z)=u_{h}\left(\frac{z}{z_{h}}\right)^{s}
$$

where $u_{p b l}$ is the prescribed boundary layer velocity at a given height $z, u_{h}$ is the reference velocity at hub height $z_{h}$, and $s$ is an empirically obtained coefficient that varies dependent on the stability of the atmosphere.

In our previous work we have shown how for simulations with a small azimuthal discretization (2.5 degrees or smaller), a first order time stepping is capable of correctly capturing the rotor and wake dynamics [17]. Since in the present work the far wake model has been considerably modified, a similar study has been carried out. A wake azimuthal discretization of 1.5 degrees has been used for the study. Simulations have been carried out using first, second and third order Runge-Kutta schemes obtaining almost identical results in terms of first order wake statistics.

\subsection{Prescribed shear}

In the following, different approaches for the modeling of a sheared inflow in vortex methods are investigated. 


\begin{tabular}{|c|c|c|c|c|}
\hline Simulation ID & Wake Elements & Wake model & Wind Shear model & Ground model \\
\hline id.01 & filaments & free-wake (hybrid) & prescribed velocity & non \\
\hline id.02 & particles & particle-mesh & prescribed velocity & non \\
\hline id.03 & particles & particle-mesh & prescribed velocity-vorticity & non \\
\hline id.04 & particles & particle-mesh & prescribed velocity-vorticity & slip wall \\
\hline
\end{tabular}

Table I. Prescribed shear simulation test cases.

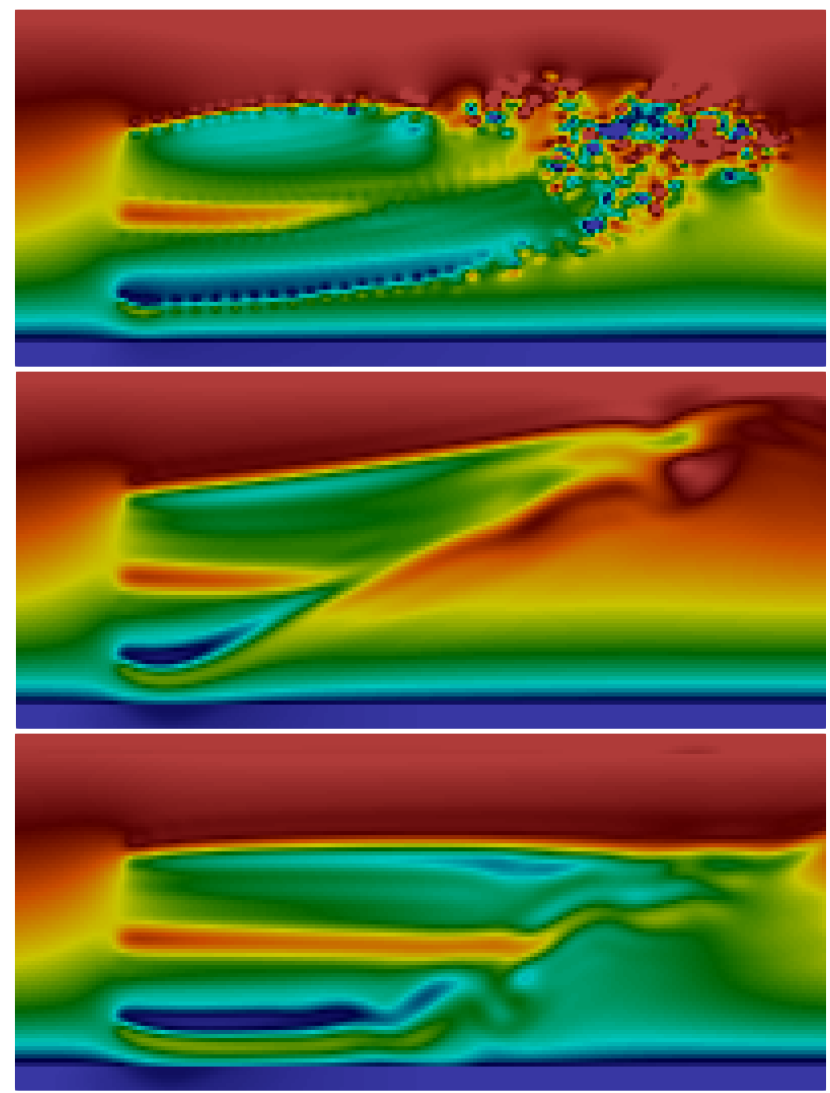

Figure 4. Iso-velocity field comparing the different wake and wind shear models (top) filament free-wake with velocity prescribed wind shear, (middle) particle wake with velocity prescribed wind shear, (bottom) particle wake with the prescribed velocity-vorticity boundary layer method (P2VBL).

A laminar inflow is used in all the cases analyzed, which are summarized in Table I. A comparison of the instantaneous stream-wise velocity field in a plane perpendicular to the ground passing through the rotor center is shown in Figure 4. The top left figure depicts the wake predicted by the filament free-wake model (id.01), an auxiliary mesh is used to speed up the simulation as in [17]. The shear model uses a prescribed velocity approach, and therefore is only accounted for by advecting the filament end markers with the height dependent stream-wise velocity. As clearly observed in the figure, the wake non-physically deflects upwards. The same occurs in the second case (id.02, middle figure). In this case, a set of particles is used to model the wake. The vorticity transport equation is solved to model the evolution of vorticity in time, including stretching and diffusion effects. In terms of the shear model, the previous prescribed velocity implementation is employed. An even more abrupt upward motion of the wake is predicted by the particle-mesh solver. The bottom figure represents the presented P2VBL method (id.03). The shear contributions to vortex stretching and convection introduced by the P2VBL model prevent the unphysical up-ward motion seen in the previous cases. The influence of the P2VBL model in the diffusion mechanisms is minimal. 


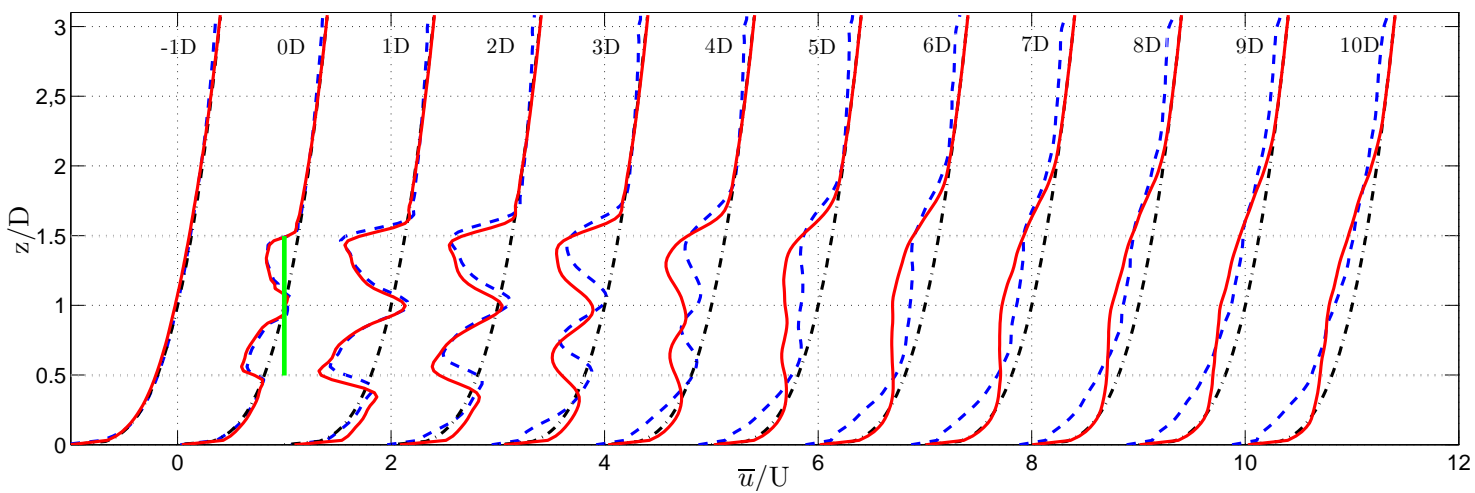

Figure 5. Averaged stream-wise velocity profiles normalized with the free-stream speed at different locations, from 1 diameter upstream to 10 diameter downstream of the rotor plane. Comparison between simulations with a slip wall BC (red solid lines) and free BC (blue dashed lines) used for the ground boundary. The dash-dotted lines represent the inflow profile.

In the vortex simulation of an atmospheric boundary layer profile, the inclusion of the ground effect is very important as shown in Figure 5. In the figure, the simulated mean velocity profiles up to 10 diameters downstream the rotor plane with and without modeling the ground are shown. In one simulation, a free boundary condition (BC) is used in all directions (id.03), while in the other, the ground is modeled using the previously introduced slip wall (id.04). The addition of the ground has two positive impacts. First, it avoids the non-physical deceleration of the flow between the lower edge of the wake and the ground, which can be seen in the free BC simulation. And second, it helps to completely correct the remaining upward motion of the wake, clearly seen between three to five diameters downstream.

Preventing the artificial upwards deflection of the wake and the flow deceleration near the ground is the first step towards a more detailed verification of the P2VBL method. Nonetheless the different wake characteristics and its downstream development need to be investigated further to obtain a better picture of the P2VBL model capabilities. This is done in Subsection 3.4.

\subsection{Turbulent inflow}

A verification of the free vorticity turbulence inflow model used in this work is presented in what follows by comparing it to the more standardized frozen velocity-based approach. A uniform inflow case with a $10 \%$ inflow turbulence is selected for the comparison. Fully aeroelastic computations with the MIRAS code are performed with a time discretization of 0.01 seconds and a total number of 72000 time steps.

In Figure 6 the power spectral density of four relevant turbine signals is shown. The top-left figure shows the power spectral density of the stream-wise velocity, with the dot-dashed line depicting the $-5 / 3$ slope, representative of the inertial sub-range in homogeneous turbulence. As seen, this is well captured by both approaches. The filtering of the high frequency fluctuations by the Poisson solver can be observed in the free vorticity method predictions. Regarding the wind turbine response, the signals for electric power, flap-wise deformation and root bending moment are presented. In general, both ways of accounting for the incoming turbulence display a similar response of the turbine. However, the frozen approach tends to predict slightly larger peaks, mostly in the high frequency range.

This paper is not focussed on the turbulence modeling approach per se, although a deeper analysis of the uniform turbulent inflow simulations would be of interest. From now on the free vorticity turbulence inflow model is used in all simulations, since it represents the only means of predicting the two-way interaction between the wind turbine, the wake and the inflow turbulence. 

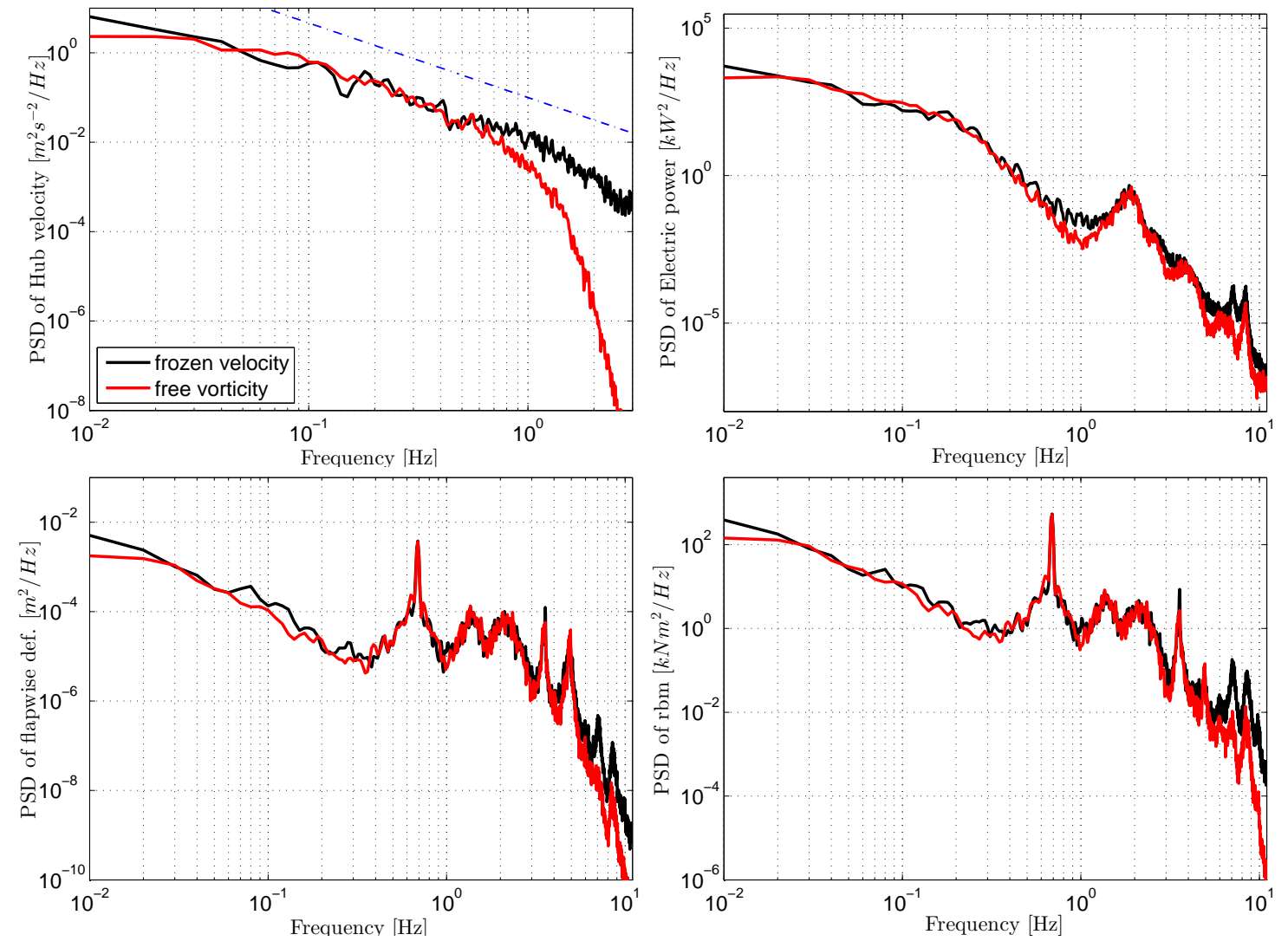

Figure 6. Power spectral density (PSD) of the (top-left) hub height velocity (top-right) electric power (bottom-left) flapwise deformation of blade one (bottom-right) root bending moment of blade one $(\mathrm{rbm})$. Comparison between the frozen and the free turbulent inflow models.

\subsection{Parametrical study}

In what follows two different parametrical studies are presented. In the first one, the ability of the P2VBL implementation to model different shear flows, from a mild to an extreme case is analyzed. In the second one, we investigate the models capability to cope with different turbulent inflow intensities.

\subsubsection{Power-law coefficient variation}

In this subsection the ability of the P2VBL model to simulate different types of shear is analyzed. Simulations for three different power law coefficients have been carried out and the results are presented in the following. The selected cases range from a mild shear, representative of a neutral stability condition in the atmosphere, $s=0.15$, to an extreme shear, $s=0.45$. A shear coefficient of $s=0.30$ is taken as representative of an intermediate case. In this subsection all cases have been simulated with a inflow turbulence intensity of $3 \%$ at a wind speed of $10.5 \mathrm{~m} / \mathrm{s}$. In Figure 7 is shown a threedimensional visualization of the instantaneous vorticity iso-contours for the mild shear case. Besides the vorticity released by the wind turbine blades, from the picture one can isolate two other sources of vorticity. One related to the incoming turbulence that appears as smaller and rounded blobs appearing randomly around the domain, and a second one related to the large velocity gradients near the ground, which generates larger and more stretched vortex structures. Visualizations of the stream-wise velocity field at the mid-vertical plane of the wake for the three shear cases are depicted in Figure 8 . From the figure it can be appreciated that increasing the shear coefficient generates more mixing in the wake.

The development of the mean non-dimensional stream-wise velocity at nine different cross sections in the wake is depicted in Figure 9. Note here that the speed-up observed around the wake center is caused by the missing tower and 


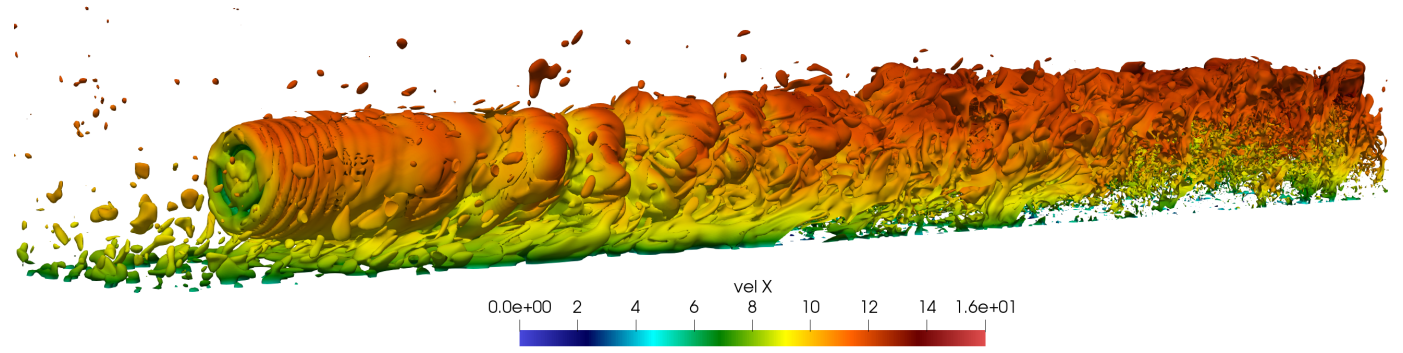

Figure 7. Visualization of the vorticiy iso-contour of the flow past the rotor at $10.5 \mathrm{~m} / \mathrm{s}$ immersed in an atmospheric boundary layer with a turbulent intensity of $3 \%$ and a power law coefficient of 0.15 . The iso-contours are colored by the magnitude of the streamwise velocity.

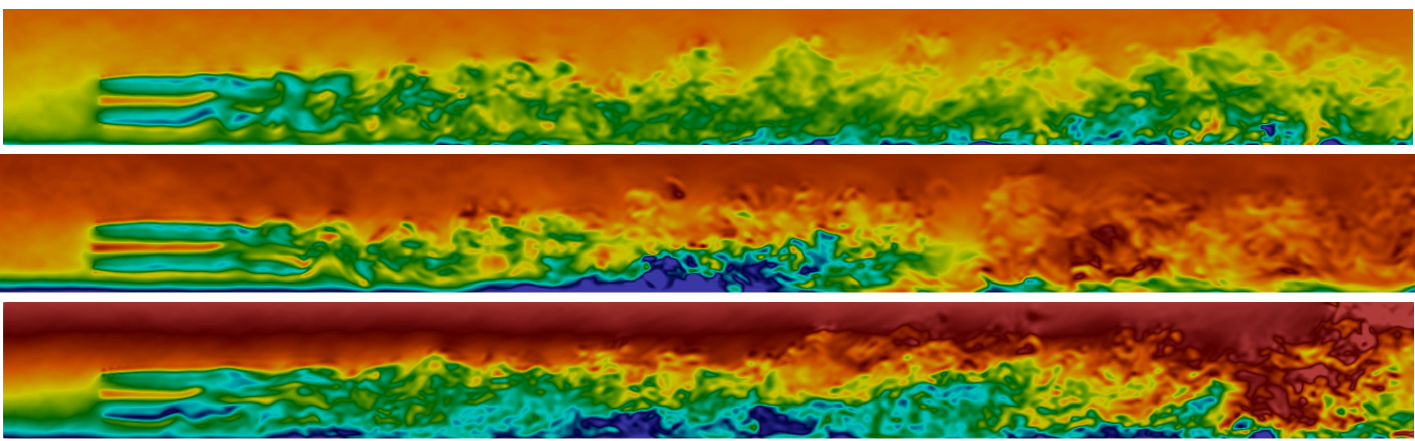

Figure 8. Visualization the stream-wise velocity in a two-dimensional cross-section of the flow past the rotor at $10.5 \mathrm{~m} / \mathrm{s}$ immersed in an atmospheric boundary layer with a turbulent intensity of $3 \%$ and a power law coefficient of (top) 0.15 (center) 0.30 and (bottom) 0.45 (the figure uses the same color-map as the previous one).

nacelle from the simulations. As reported in [34], this effect lasts up to 5 diameters behind the rotor. An acceleration of the flow between the ground and the lower edge of the wake is predicted in the "near wake" region (at 2 and 3 diameters downwind locations). This effect increases with increasing shear coefficient. As expected, at the far downstream positions the turbine influence in the mean velocity profile almost vanishes, and the predicted boundary profile resembles the incoming one measured 1 diameter upstream of the rotor plane, attaining an almost-complete wake recovery.

Figure 10 shows the velocity deficit, the turbulence intensity, and Reynolds stresses, respectively, at three different cross sections. From the averaged wake velocity deficit depicted in Figure 10 (left column) its observed that the velocity distribution for the sheared case is not initially axisymmetric (up to 5 diameters downwind). However, as the wake recovers further downstream, axisymmetry appears. This can be seen at the 7 diameters location, where the wake presents a well defined Gaussian shape, very similar to a uniform inflow case. In the middle column of Figure 10 its shown how the turbine enhances the turbulent intensity downwind of the rotor plane, with the highest values reached around the tip region. The simulations show a non-axysimmetric distribution of the turbulence intensity, caused by the influence of the atmospheric boundary layer, as previously found experimentally [21]. Between 2 to 10 diameters downstream of the turbine, there is a strong enhancement of the turbulent intensity in the top part of the wake $(x \approx 1.5 D)$ for the sheared simulations due to the combined effect of the shear and the helicoidal tip vortices. A remarkable change in the turbulent intensity distribution is observed further downstream. Turbulent enhancement in the upper part of the rotor is reduced, increasing in the region below the hub height. The non-dimensional distribution of the cross-sectional Reynolds stresses $\overline{u^{\prime} w^{\prime}}$ shown in Figure 10 (right column) gives insight in the turbulent momentum transfer in the flow. As previously reported by [21], there is a region below the wind turbine hub with a large positive stress and another region above the hub height with a minor negative stress (distances up to 3 diameters downstream). With the further downstream development of the wake, the magnitude of the stress peak is reduced in the lower region, while it increases above the hub height. The negative peak seen 7 diameters downstream of the turbine can be related to the re-energization of the boundary layer with flow entering 

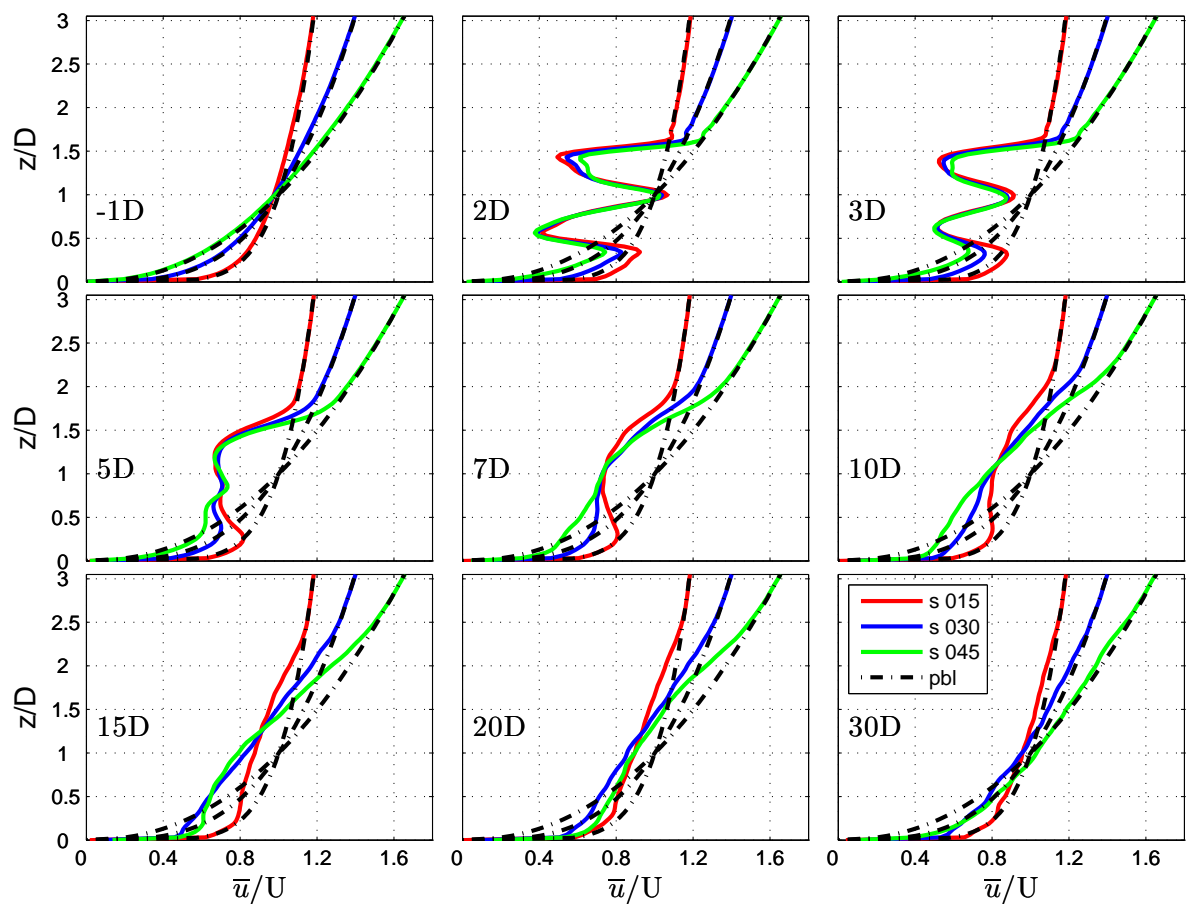

Figure 9. Non-dimensional distribution of the stream-wise velocity at different downstream locations, comparison between the power law shear coefficients of $0.15,0.30$ and 0.45

the wake from its upper limit (negative $w$-component) due to the effect of the tip vortex. Further downstream the Reynolds stress distribution develops further, slowly reducing its magnitude in the upper region and slightly increasing it in the lower one. This can be related to the large gradients in the stream-wise velocity near the ground, which seems to be the main driver of the $\overline{u^{\prime} w^{\prime}}$ cross-sectional distribution far downstream. Promoting the upward momentum transfer from the lower regions of the boundary layer towards the center of the wake. Comparing the different shear cases, it is observed that in the near wake locations the Reynolds stresses magnitude is smaller for the extreme shear case. However, as the wake develops this tendency is clearly inverted, indicating that the larger the shear, the more mixing exists. Especially from structures entering the wake from its upper edge. The distribution of the turbulent intensity terms shows a similar picture.

In what follows we study the impact of the simulated shear profiles in the aeroelastic turbine response. A time representation of such response is shown in Figure 11. Three sensors are analyzed, i.e. electric power, rotor thrust and flap-wise deflection on one blade. Predictions show a $1 \%$ increase in the average generated power for the 0.45 shear case in comparison with the 0.15 case, and a $0.35 \%$ increase for the 0.30 case. This increase is an order of magnitude larger than the one obtained from BEM predictions [35]. Such discrepancy is due to the herein predicted acceleration of the flow between the ground and the lower edge of the turbine, which is not accounted for in BEM codes. In terms of thrust force, an insignificant variation of the mean value is predicted by the vortex solver. However, there is a small amplification of the high frequency oscillations. Regarding the blade deflections, the amplitude of the 1P oscillations increases drastically with the increasing shear coefficient. Increments in the deflections of up to $15 \%$ respect to the mild shear are observed for the extreme case. For the medium shear case there is an increase of up to $6 \%$. As expected, the influence of shear is mostly concentrated in the blade loading and not in the power production nor rotor loading. A spectral analysis of the previous signals is not presented in the manuscript since in this case the time signals show a better picture of the effect of shear.

\subsubsection{Turbulence intensity variation}

An analysis of the influence of the turbulent intensity in the simulations is presented in this section. The Mann turbulence model [15] is used to generate a turbulent wind field, with magnitude of the fluctuations scaled to obtain three turbulence 

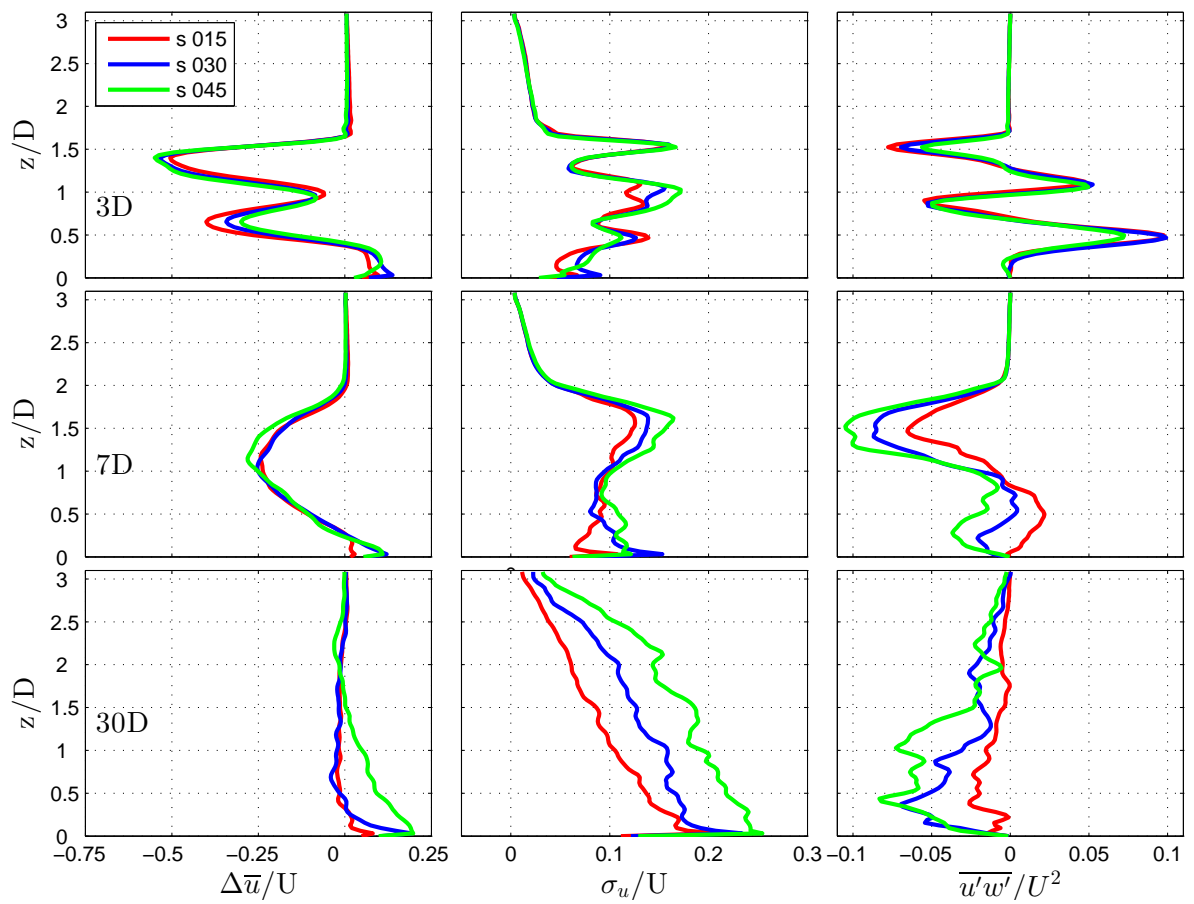

Figure 10. Non-dimensional distribution of wake quantities at 3, 7 and 30 diameters downstream the rotor plane (left) velocity deficit (center) turbulent intensity and (right) cross-sectional Reynolds stresses, comparison between the power law shear coefficients of $0.15,0.30$ and 0.45 .

intensity cases of $1 \%, 3 \%$ and $10 \%$. A power law shear coefficient of 0.30 is used in all cases with an incoming wind speed of $10.5 \mathrm{~m} / \mathrm{s}$. Figure 12 shows a visualization of the vorticity iso-contours for $\mathrm{TI}=10 \%$. A snapshot of the stream-wise velocity field on a mid-vertical plane along the wake is presented in Figure 13 for all three cases. It can be observed how an increasing turbulent intensity tends to break the coherent wake structures breaks into turbulence closer to the rotor plane. This creates a less stable wake and increases the mixing of the top layers of the boundary layer with the ambient turbulence.

The spatial distribution of the averaged stream-wise velocity at the mid-vertical plane of the wake is shown in Figure 14. It is clear from the figure that a higher the turbulence intensity faster recovers the boundary layer towards its original shape. The flow acceleration due the missing tower and nacelle is reduced with the increasing turbulence level.

The averaged quantities of the velocity deficit, local turbulent intensity, and the $\overline{u^{\prime} w^{\prime}}$ Reynolds stresses for the locations of 3, 7 and 30 diameters downstream the rotor plane are shown in Figure 15. As reported in [22], the wind turbine enhancement of the turbulent velocity fluctuations increases with the increasing TI, generating more turbulent mixing in the wake. As we move downstream, the differences between the three cases are considerably reduced and the turbulent mixing becomes larger near the ground, where the effect of the shear is stronger. In terms of $\overline{u^{\prime} w^{\prime}}$ Reynolds stresses, the shear effect seems to be masked by the effect of the incoming turbulent intensity in the near wake region. With the peak strength being inverted for the high TI case in comparison with the lower ones, showing a larger downwards momentum transfer. Opposite to the low TI case, where the shear dominates and the upwards momentum transfer is larger.

The time representation of the turbine aeroelastic response to the different TI is shown in Figure 16. Opposite to the influence of shear, and due to the randomness of turbulence, the TI affects in a similar way both the turbine loading and the local blade loading. For a better understanding of the turbine response, the power spectra density of the previous signals are depicted in Figure 17. A similar picture is seen in the frequency domain for the power and thrust signals. It is observed how only the high TI manages to penetrate the mid and high frequency response of the power output. In terms of blade deflections, the energy level of the $1 \mathrm{P}$ and $2 \mathrm{P}$ frequency peaks is maintained independently of the TI. This is an 

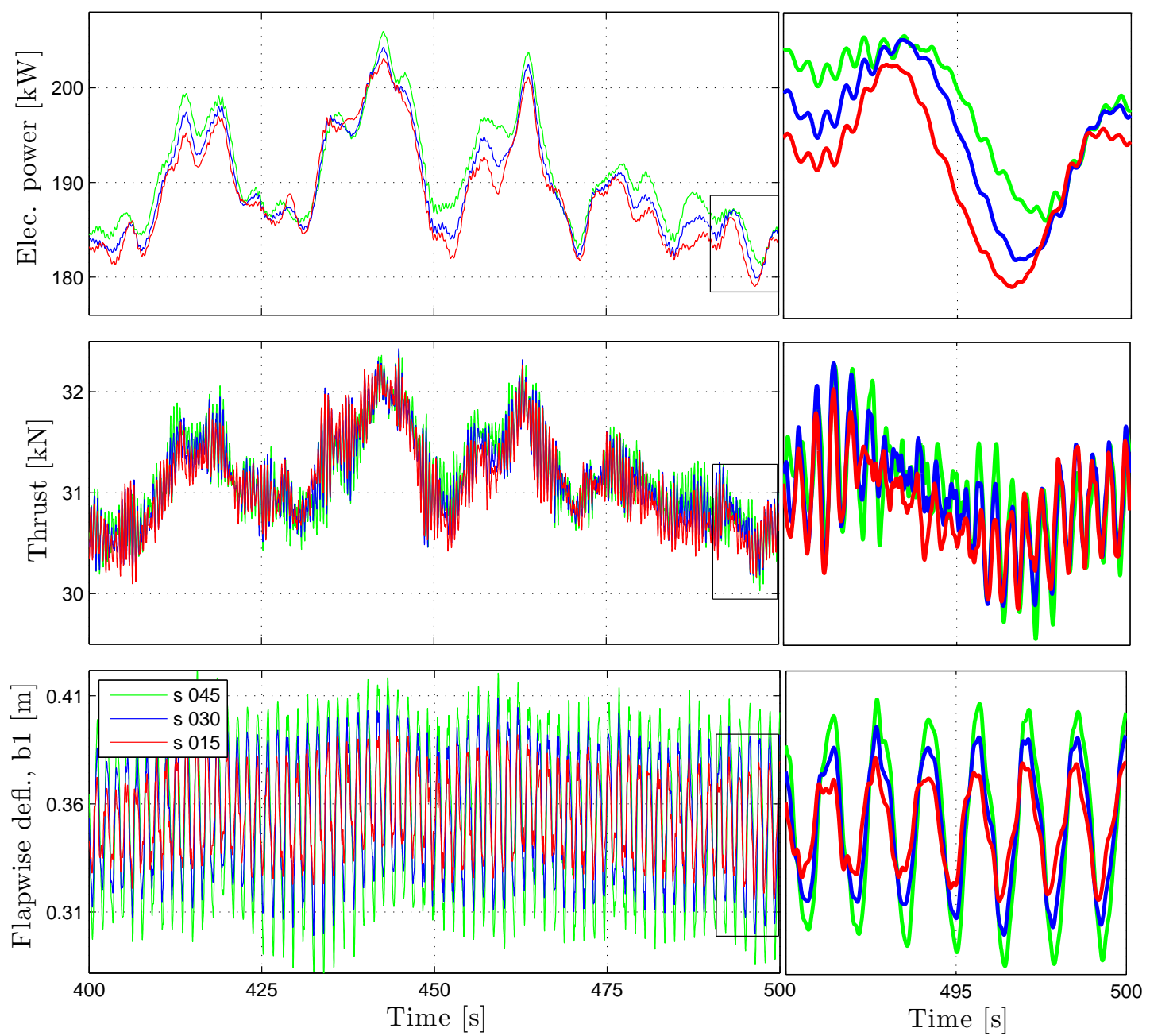

Figure 11. Time signal of (top) electric power (center) thrust force (bottom) flapwise deflection on blade one. The images to the left display the last 100 seconds of the simulations and a zoom into the last 10 seconds is shown to the right. Comparison between the power law shear coefficients of $0.15,0.30$ and 0.45 intensities.

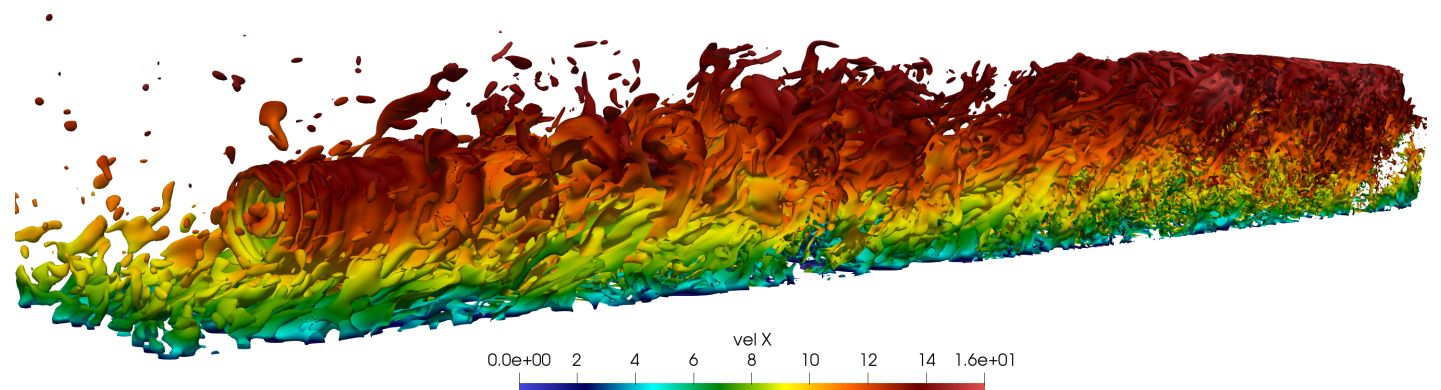

Figure 12. Visualization of the vorticiy iso-contour of the flow past the rotor at $10.5 \mathrm{~m} / \mathrm{s} \mathrm{immersed} \mathrm{in} \mathrm{an} \mathrm{atmospheric} \mathrm{boundary} \mathrm{layer}$ with a turbulent intensity of $10 \%$ and a power law coefficient of 0.30 . The iso-contours are colored by the magnitude of the streamwise velocity.

indication that the main flow is the responsible of such frequency peaks, while the turbulent structures mostly affect the higher harmonics in the blades. 

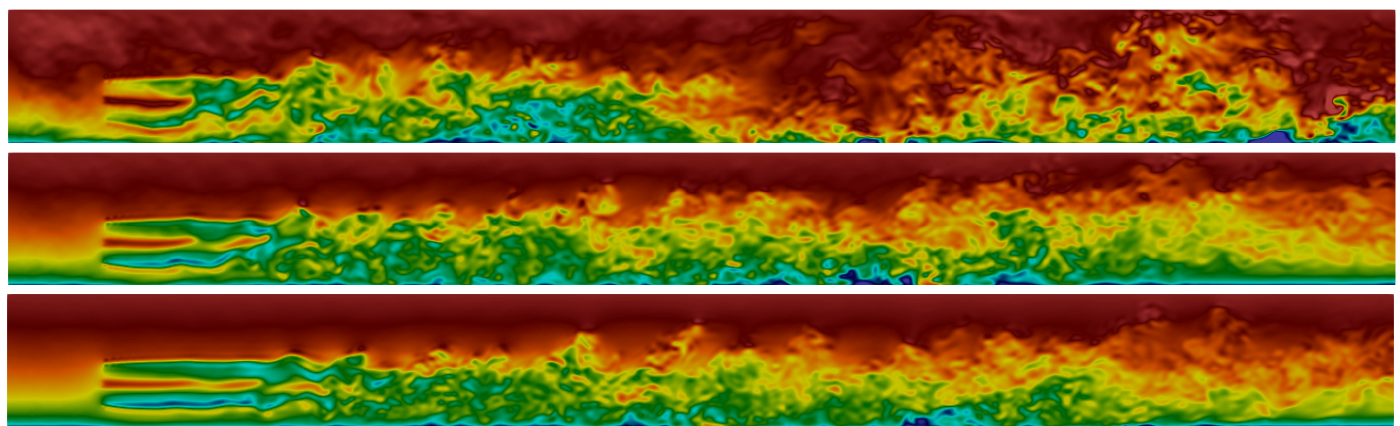

Figure 13. Visualization the stream-wise velocity in a two-dimensional cross-section of the flow past the rotor at $10.5 \mathrm{~m} / \mathrm{s}$ immersed in an atmospheric boundary layer with a turbulent intensity of (top) $10 \%$, (center) $3 \%$ and (bottom) $1 \%$ and a power law coefficient of 0.30 (same color-map as previous figure).
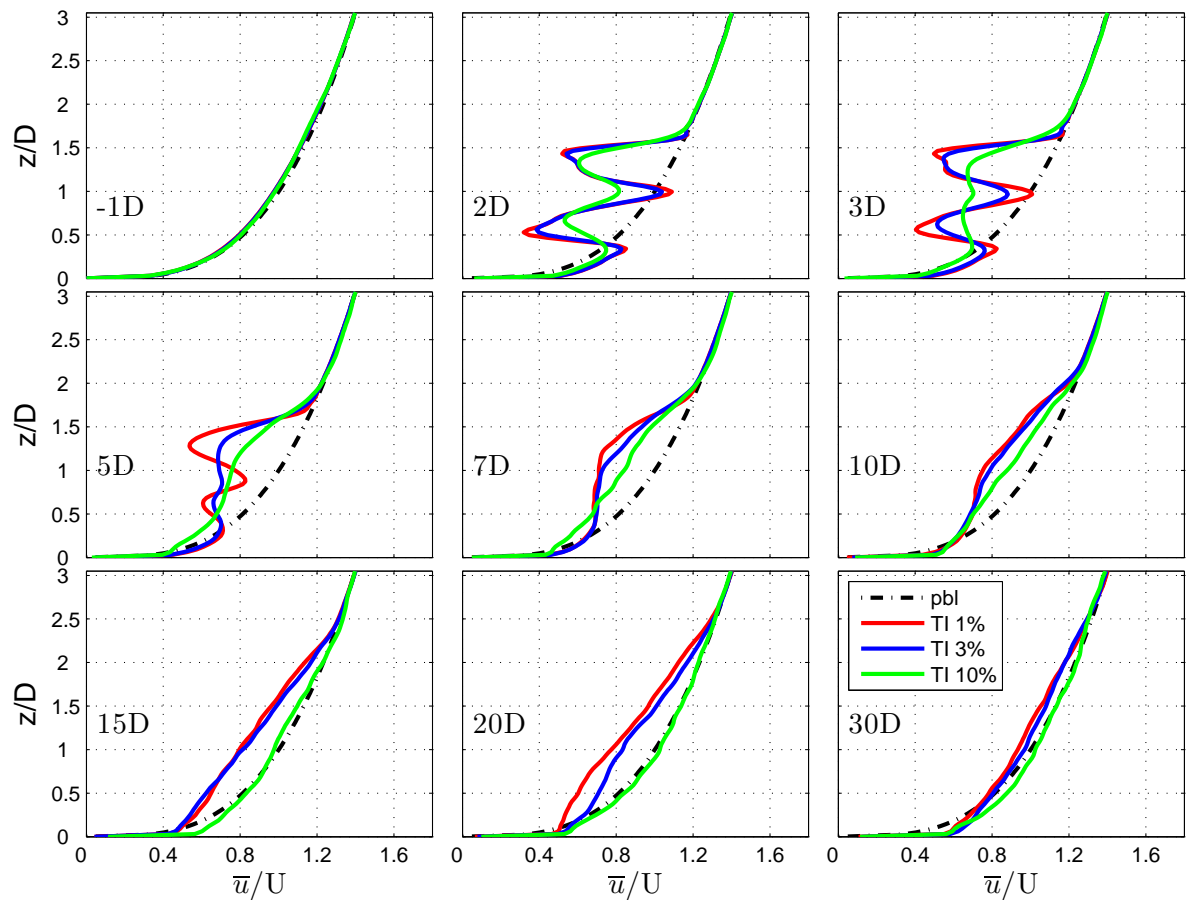

Figure 14. Non-dimensional distribution of the stream-wise velocity at different downstream locations, comparison between different $\mathrm{TI}$ and the prescribed boundary layer for the measured profile case

The power spectral density of the stream-wise velocity signal one diameter behind the wind turbine at three different heights of $1.6 z_{t i p}, 1.0 z_{t i p}$ and $0.75 z_{t i p}$ is shown in Figure 18. Here $z_{t i p}$ refers to the blade tip height. As seen in the figure, the higher the turbulent intensity the more energy is contained in the velocity spectrum. It is also interesting to note here the signature of the tip vortices, clearly seen in the middle figure. With a peak appearing at a frequency three times larger than that of the turbine (3P), around $2.05 \mathrm{~Hz}$ (value not exact due to the action of the turbine controller). It is observed how the tip vortex strength weakens as the turbulent intensity increases. 

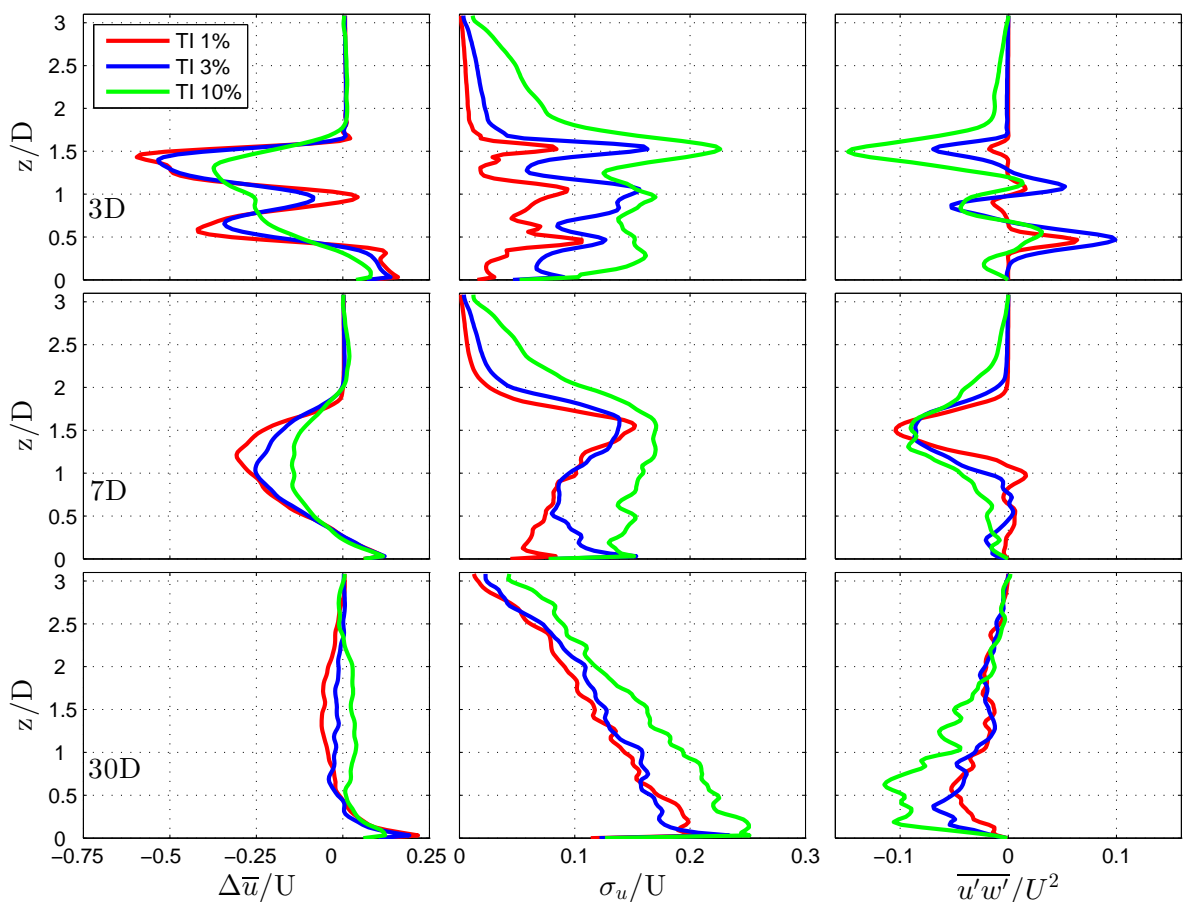

Figure 15. Non-dimensional distribution of wake quantities at 3, 7 and 30 diameters downstream of the rotor plane velocity deficit (left), turbulent intensity (center) and cross-sectional Reynolds stresses (right), comparison for turbulent intensities of $1 \%, 3 \%$ and $10 \%$.

\section{CONCLUSION}

A model for representing the atmospheric boundary layer in vortex simulations of wind turbine wakes has been presented. The method uses a prescribed velocity-vorticity implementation of the shear terms in the vorticity transport equation with a slip wall boundary condition to model the ground. A Lagrangian implementation is proposed and examined in detail.

Initially a comparison between the different methods for imposing a shear flow is carried out. The proposed model shows a clear advantage in comparison with the generally used velocity prescribed approach, correcting the unphysical upwards deflection of the wake seen in previous work. It has been found that the correct modelling of the ground is important to obtain a well developed wake far downstream the rotor plane. A short investigation regarding the inflow turbulence modeling is performed. An advanced free vorticity modeling of the turbulence, represented by a set of upstream released vortex particles, results in a very similar turbine response in comparison with a simpler frozen velocity approach. However, in the free approach the interaction between the inflow turbulence and the wake is taken into account, which is of key importance for the further study of a turbine immersed in an atmospheric boundary layer flow.

The capability of the velocity-vorticity boundary layer method (P2VBL) to correctly represent an atmospheric boundary layer is assessed by carrying out two different studies. The first study analyzes the influence of the type of shear, where three different cases, ranging from mild to extreme shear, are analyzed. In the second study the influence of the incoming turbulent intensity is analyzed. Both studies present a detailed investigation of averaged wake quantities, such as velocity deficit, turbulent intensity and Reynolds stresses, showing a good qualitative agreement of the predicted behavior with findings from previous experimental and numerical work performed on small size rotors.

The present work aims to be a proof of concept of the P2VBL model and therefore quantitative comparison with experimental work is not included. Future work will focus on validating the model against experiments carried out under controlled conditions. 

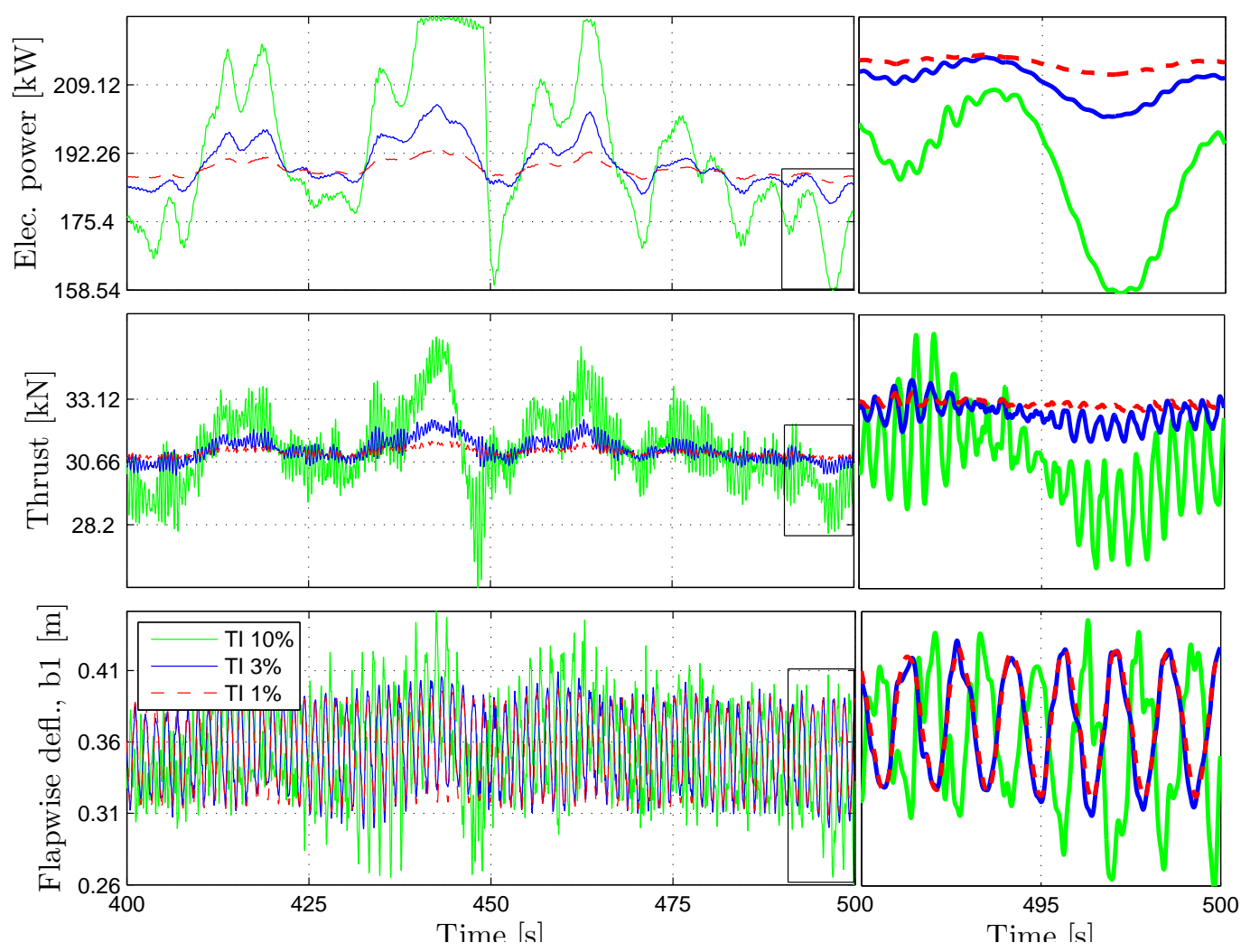

Figure 16. Time signal of electric power (top), thrust force (center) and flapwise deflection on blade 1 (bottom). The images to the left display the last 100 seconds of the simulations and a zoom into the last 10 seconds is shown to the right. Predictions for the three different turbulent intensities are included.
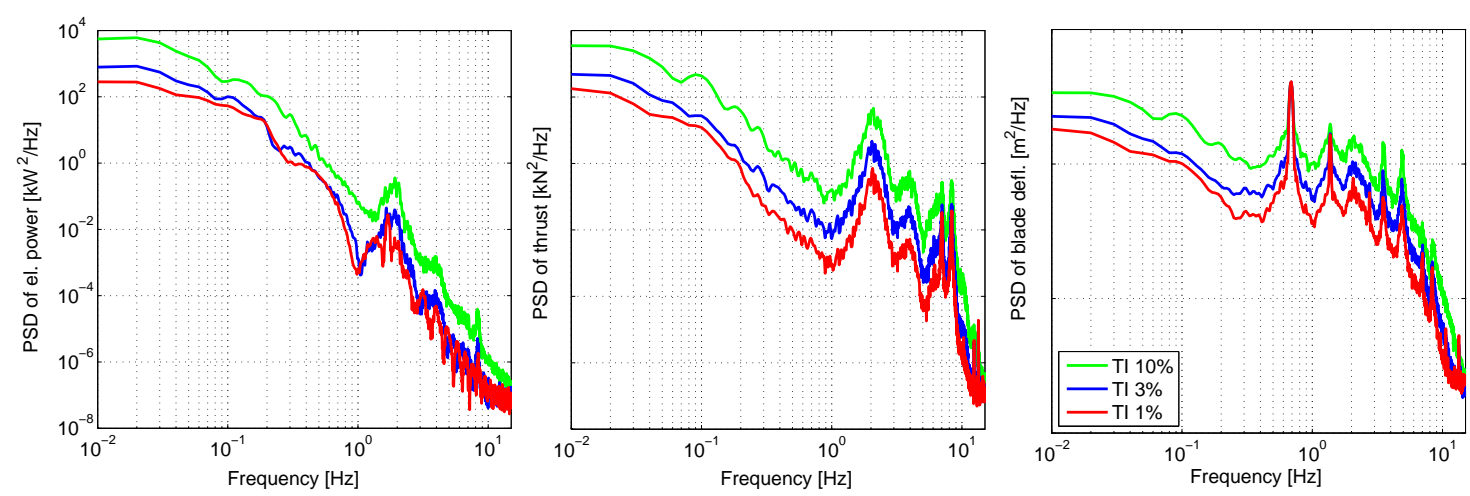

Figure 17. Power spectral density of electric power(left), thrust force (middle) and flapwise deflection on blade 1 (right). Comparison between three different turbulent intensities.

\section{ACKNOWLEDGEMENTS}

The authors would like to acknowledge the support from the European Union's Seventh Programme for research, technological development and demonstration for the project 'AVATAR: AdVanced Aerodynamic Tools for 1Arge Rotors'(FP7-ENERGY-2013-1/no. 608396). Henrik Juul Spietz is funded by the Danish Council for Independent Research grant no. 4184-00349B. 

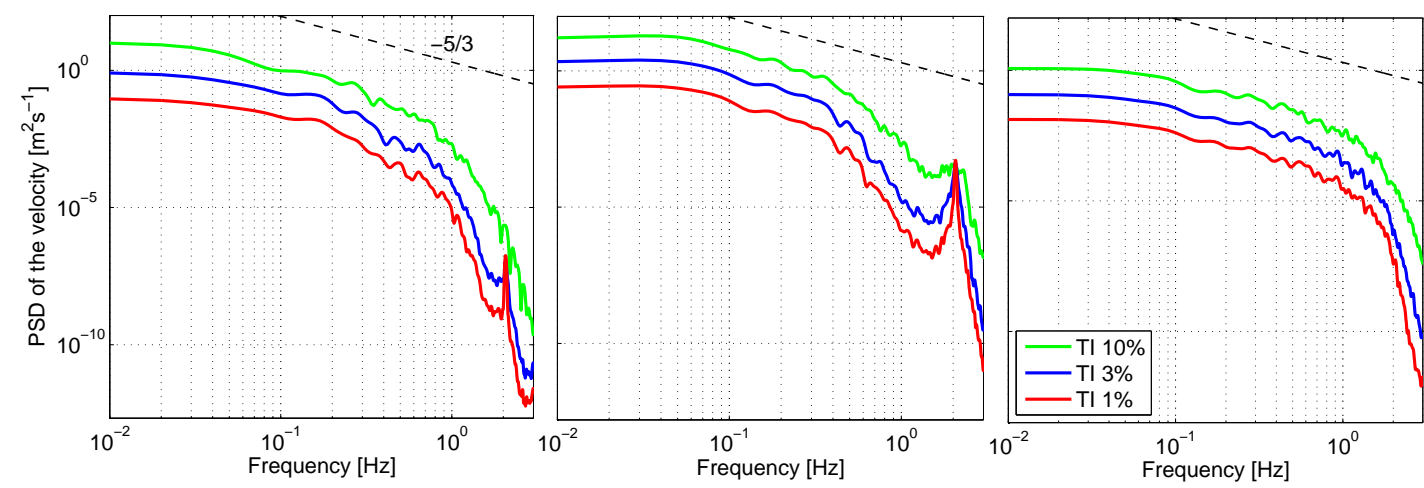

Figure 18. Filtered energy spectra of the streamwise velocity at a location one diameter downstream the rotor plane at three boundary layer heights $0.75 z_{t i p}$ (left), $1.0 z_{t i p}$ (middle) and $1.6 z_{t i p}$ (right). Comparison between the different turbulent intensities. The dashed line represents the $-5 / 3$ slope in the inertial subrange.

\section{REFERENCES}

[1] P. Chatelain, S. Backaert, L. Bricteux, and P. Koumoutsakos, "Vortex particle-mesh methods with immersed lifting lines applied to the large eddy simulation of wind turbine wakes," Wake Conference, Gotland, 2011.

[2] P. Chatelain, S. Backaert, G. Winckelmans, and S. Kern, "Large eddy simulation of wind turbine wakes," Flow Turbulence Combust., vol. 91, pp. 587-605, 2013.

[3] S. Backaert, P. Chatelain, and G. Winckelmans, "Vortex particle-mesh with immersed lifting lines for aerospace and wind engineering," IUTAM Symposium on Particle Methods in Fluid Dynamics, vol. 18, pp. 1-7, 2015.

[4] S. G. Voutsinas, M. A. Belessis, and S. Huberson, "Dynamic inflow effects and vortex particle methods," European community wind energy conference, 8-12 March, Lübeck-Travemünde, Germany., 1993.

[5] M. Sessarego, N. Ramos-García, J. Sørensen, and W. Shen, "Development of an aeroelastic code based on threedimensional viscousinviscid method for wind turbine computations," Wind Energy, vol. 20, no. 7, pp. 1145-1170, 2017.

[6] J. H. Walther, M. Guénot, E. Machefaux, J. T. Rasmussen, P. Chatelain, V. L. Okulov, J. N. Sørensen, M. Bergdorf, and P. Koumoutsakos, "A numerical study of the stabilitiy of helical vortices using vortex methods," J. Phys.: Conf. Ser., vol. 75, p. 012034, 2007.

[7] M. O. L. Hansen., Aerodynamics of Wind Turbines. Earthscan, second edition, 2008.

[8] G. Schepers, “Advanced aerodynamic tools for large rotors," http://www.eera-avatar.eu, 2017.

[9] N. Sezer-Uzol and O. Uzol, "Effect of steady and transient wind shear on the wake structure and performance of a horizontal axis wind turbine rotor," 47th AIAA Aerospace Sciences Meeting Including The New Horizons Forum and Aerospace Exposition 5 - 8 January 2009, Orlando, Florida, pp. 1-10, 2009.

[10] E. Branlard, M. Papadakis, G.and Gaunaa, G. Winckelmans, and T. Larsen, "Aeroelastic large eddy simulations using vortex methods: unfrozen turbulent and sheared inflow," J. Phys.: Conference Series, vol. 625, 2015. Wake Conference.

[11] N. Troldborg, J. N. Sørensen, R. Mikkelsen, and N. N. Sørensen, "A simple atmospheric boundary layer model applied to large eddy simulations of wind turbine wakes," Wind Energy, vol. 17, no. 4, pp. 657-669, 2014.

[12] S. Andersen, J. Sørensen, and R. Mikkelsen, "Performance and equivalent loads of wind turbines in large wind farms," Wake Conference 2017, vol. 854, 2017. 
[13] S. Andersen, J. N. Sørensen, and R. Mikkelsen, "Turbulence and entrainment length scales in large wind farms," Philosophical Transactions of The Royal Society A Mathematical Physical and Engineering Sciences, vol. 375, 2017.

[14] C. Hasager, N. Nygaard, P. Volker, I. Karagali, S. Andersen, and J. Badger, "Wind farm wake: The 2016 horns rev photo case," Energies, vol. 10, no. 3, 2017.

[15] J. Mann, “Wind field simulation,” Probab. Eng. Mech., vol. 13, no. 4, pp. 269-282, 1998.

[16] J. T. Rasmussen and J. H. Walther, Particle Methods in Bluff Body Aerodynamics. PhD thesis, DTU Mechanical Engineering, 2011. The Ph.D. Project was funded by the Danish Research Council of Independent Research (Grant. No. 274-08-0258).

[17] N. Ramos-García, M. M. Hejlesen, J. H. Walther, and J. N. Sørensen, "Hybrid vortex simulations of wind turbines using a three-dimensional viscousinviscid panel method," Wind Energy, vol. 20, p. 18711889, 2017.

[18] M. M. Hejlesen, J. T. Rasmussen, P. Chatelain, and J. H. Walther, "A high order solver for the unbounded Poisson equation," J. Comput. Phys., vol. 252, pp. 458-467, 2013.

[19] M. M. Hejlesen, T. Rasmussen, Johannes, P. Chatelain, and J. H. Walther, "High order Poisson solver for unbounded flows," Procedia IUTAM, vol. 18, pp. 56-65, 2015.

[20] M. M. Hejlesen, A high order regularisation method for solving the Poisson equation and selected applications using vortex methods. PhD thesis, Technical University of Denmark, February 2016.

[21] L. P. Chamorro and F. Porté-Agel, "A wind-tunnel investigation of wind-turbine wakes: Boundary-layer turbulence effects,” Boundary-Layer Meteorology, vol. 132, pp. 129-149, 2009.

[22] E. Barlas, S. Buckingham, and J. van Beeck, "Roughness effects on wind-turbine wake dynamics in a boundary-layer wind tunnel," Boundary-Layer Meteorology, vol. 158, pp. 27-42, 2016.

[23] R. W. Hockney and J. W. Eastwood, Computer Simulation Using Particles. Institute of Physics Publishing, Bristol, PA, USA, 2. ed., 1988.

[24] M. P. Scully, "Computation of helicopter rotor wake geometry and its influence on rotor harmonic airloads," Ph.D. Thesis, Massachusetts Institute of Technology. Dept. of Aeronautics and Astronautics, 1975.

[25] P. Koumoutsakos, A. Leonard, and F. Pépin, "Boundary conditions for viscous vortex methods," J. Comput. Phys., vol. 113, no. 1, pp. 52-61, 1994.

[26] P. Ploumhans and G. S. Winckelmans, "Vortex methods for high-resolution simulations of viscous flow past bluff bodies of general geometry," J. Comput. Phys., vol. 165, pp. 354-406, 2000.

[27] P. Ploumhans, G. S. Winckelmans, J. K. Salmon, A. Leonard, and M. S. Warren, "Vortex methods for direct numerical simulation of three-dimensional bluff body flows: Applications to the sphere at Re = 300, 500 and 1000," J. Comput. Phys., vol. 178, pp. 427-463, 2002.

[28] J. Mathew, R. Lechner, H. Foysi, J. Sesterhenn, and R. Friedrich, "An explicit filtering method for large eddy simulation of compressible flows," Phys. Fluids, vol. 15, no. 8, pp. 2279-2289, 2003.

[29] S. Stolz and N. A. Adams, "An approximate deconvolution procedure for large-eddy simulation," Phys. Fluids, vol. 11, no. 7, pp. 1699-1701, 1999.

[30] S. Stolz, N. A. Adams, and L. Kleiser, "An approximate deconvolution model for large-eddy simulation with application to incompressible wall-bounded flows," Phys. Fluids, vol. 13, no. 4, pp. 997-1015, 2001.

[31] J. Prendergast, Simulation of unsteady 2-D wind by a vortex method. Ph.D. thesis, Department of Engineering. University of Cambridge, 2007. 
[32] J. T. Rasmussen, M. M. Hejlesen, A. Larsen, and J. H. Walther, "Discrete vortex method simulations of the aerodynamic admittance in bridge aerodynamics," JWEIA, vol. 98, pp. 754-766, 2010.

[33] M. M. Hejlesen, J. T. Rasmussen, A. Larsen, and J. H. Walther, "On estimating the aerodynamic admittance of bridge sections by a mesh-free vortex method," JWEIA, vol. 146, pp. 117-127, 2015.

[34] R. Stevens, L. Martnez-Tossas, and C. Meneveau, "Comparison of wind farm large eddy simulations using actuator disk and actuator line models with wind tunnel experiments," Renewable Energy, vol. 116, pp. 470-478, 2018.

[35] B. J. Gould and D. L. Burris, "Effects of wind shear on wind turbine rotor loads and planetary bearing reliability," Wind Energy, vol. 19, pp. 1011-1021, 62016. 